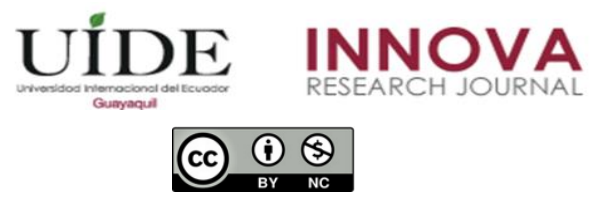

INNOVA Research Journal, ISSN 2477-9024

(Septiembre - diciembre 2019). Vol. 4, No.3 pp. 185-192

DOI: https://doi.org/10.33890/innova.v4.n3.2019.1026

URL: http://revistas.uide.edu.ec/index.php/innova/index

Correo: innova@uide.edu.ec

\title{
Influencia del Mapeo de Objeto Relacional en el rendimiento académico aplicado a proyectos en instituciones de educación superior
}

\section{Object Relational Mapping Influence on academic performance applied to projects in higher education institutions}

\author{
Orlen Ismael Araujo Sandoval \\ Universidad Laica Eloy Alfaro de Manabí, Ecuador
}

Autor para correspondencia: orlenisma@hotmail.com

Fecha de recepción: 18 de junio del 2019 - Fecha de aceptación: 29 de agosto del 2019

\begin{abstract}
Resumen
La educación se ha visto beneficiada con la aplicación de las tecnologías de la información y comunicación (TIC) al crear espacios de aprendizaje colaborativo denominados entornos virtuales de aprendizaje (EVA) en donde el estudiantado puede compartir sus opiniones y revisar contenidos académicos, sin embargo, no siempre se obtiene el desempeño académico deseado. La presente investigación pretende determinar que framework de mapeo de objeto relacional (ORM) puede ser aplicado como una herramienta de enseñanza de programación web que permita alcanzar un óptimo desempeño académico del estudiantado. Por lo antes mencionado se aplica el estudio a 92 estudiantes de la carrera de sistemas entre los periodos académicos abril - septiembre 2018 y octubre 2018 - febrero 2019 en la Universidad Laica Eloy Alfaro de Manabí extensión en El Carmen. Se utilizó una metodología cuantitativa en la evaluación de un sistema de control de inventario y la valoración que se les dio a los framework ORM utilizados. Se aplicó una encuesta cuya tabulación fue realizada con herramientas especializadas para interpretación de los datos, con ello se pudo conocer que Zend es el framework con mayor deserción.
\end{abstract}

Palabras Claves: framework; mapeo objeto relacional; laravel; zend; yii

\begin{abstract}
The Education has benefited from the application of information and communication technologies (ICT) by creating collaborative learning spaces called virtual learning environments (EVA) where the student can share their opinions and academic content, however, The desired academic performance is not always obtained. The present investigation tries to determine that framework of relational object mapping (ORM) can be applied as a teaching tool of web programming that allows to reach an excellent academic performance of students. For the study is applied to 92 students of the systems career between the academic periods April - September 2018 and October 2018 - February 2019 at the Eloy Alfaro de Manabí extension in El Carmen University. A quantitative methodology was considered in the evaluation of an inventory control system and the valuation that was given an ORM framework used. A survey was applied whose tabulation was carried out with specialized tools for interpretation of the data; with this, it was known that Zend is the framework with the highest dropout
\end{abstract}

Key words: framework; object relational mapping; laravel, zend, yii 


\section{Introducción}

En la actualidad la prominente vinculación de las Tecnologías de la información y la comunicación (TIC) han presentado un gran avance en la educación, al dejar de lado a la metodología tradicional que de acuerdo con Bonvecchio (2006) es una estrategia que tiene como base el suponer que la enseñanza se transmite solo en la palabra, es decir, el docente explica y el alumno solo escucha mas no opina. Esta ideología se desplaza ya que las TIC permiten acceder a fuentes de información masiva, por lo cual el estudiante debe aprender a escuchar de forma crítica, analizar y compartir ideas u opiniones con otras personas.

La presenta investigación realiza un análisis comparativo entre tres framework, con el objetivo de determinar cuál de ellos puede utilizarse como una herramienta de enseñanza en la catedra de programación web y a través de esta alcanzar un óptimo rendimiento académico de los estudiantes. Se tendrá en consideración el cumplimiento de los requerimientos y el promedio académico al término del desarrollo de un software de control de inventario.

\section{Las Tecnologías de la Información en la Educación}

La utilización de las TIC en el sector educativo han demostrado efectividad al fortalecer la capacidad intelectual de los estudiantes, al convertirse en un medio que contribuye con el desarrollo cognitivo e integral del estudiante (Mendoza, 2018), en donde de acuerdo con Centeno Herrara (2018) el desarrollo integral se conforma por el modelo de enseñanza, desarrollo humano y práctica profesional, que, al ser potencializados y trabajados de forma conjunta, posibilitan en el estudiante el reconocimiento de sus fortalezas y debilidades.

EL fortalecimiento de la educación a través de las TIC ha originado nuevas metodologías de enseñanza como el Blended Learning (B-Learning) que de acuerdo con Dionicio (2014) este método está fuertemente marcado por el trabajo autónomo que realiza el estudiante a través de los entornos virtuales de aprendizaje (EVA) que se combinan con la catedra presencial. Los EVA se muestran como una herramienta informática que permite la difusión del material pedagógico, recursos multimedia y la comunicación directa entre docente y estudiante, además, proporciona un área de retroalimentación del trabajo que realiza el alumno con el fin de que este mejore progresivamente. La fusión de un entorno virtual y presencial crea un espacio de aprendizaje colaborativo en donde la interacción del grupo de estudio proporciona un refuerzo del conocimiento adquirido, esto a través de medios síncronos o asíncronos que garantizan la asimilación del conocimiento y acreditan un alto rendimiento académico. (Orozco, y otros, 2017) (Ruiz \& Aguirre, 2013).

El rendimiento académico se asocia con el nivel de conocimiento y habilidades que un alumno ha obtenido durante su vida estudiantil y ha desarrollado a través de instrumentos de evaluación, con ello se determina las capacidades que cada alumno ha obtenido durante el proceso formativo e identifica las debilidades o temáticas a fortalecer para evitar vacíos de conocimiento (Costa, 2015). Los instrumentos de evaluación no pueden utilizarse de forma generalizada, es así como, en carreras tecnológicas estos varían, ya que evalúan el conocimiento adquirido a través del desarrollo de herramientas tecnológicas ya sean estas aplicaciones o sistemas de información en las que interviene la ingeniería de sistemas. 


\section{Ingeniería de Software}

De acuerdo con Pantaleo y Rinaudo (2015) la ingeniería de software es la disciplina que se encarga de estudiar el desarrollo, operación y mantenimiento de software, cuya práctica académica se centra en las dos primeras etapas. Durante el desarrollo de software es importante seleccionar una correcta metodología que determine el proceso a seguir y evite exceder los tiempos estimados, esto es complementado con herramientas que un usuario puede aplicar en su vida profesional para satisfacer los requerimientos tecnológicos en una empresa u organización.

Dentro de la ingeniería de software se establecieron las metodologías tradicionales, las cuales imponen una disciplina durante el proceso de desarrollo de software al enfatizar la planificación a detalle de cada etapa, esto conlleva una mayor inversión tiempo y una reducción en la capacidad de adaptación a cambio de requerimientos sobre el sistema. A diferencia las metodologías ágiles surgen como una alternativa, este tipo de metodologías buscan reducir el tiempo de desarrollo del aplicativo al trabajar de forma colaborativa mediante técnicas y herramientas que promueven la flexibilidad y adaptabilidad a cambios en los requerimientos. Las actualizaciones son realizadas con prontitud ya que mantienen una relación cercana con el cliente final, lo que facilita prever las modificaciones y cumplir los requisitos de software de forma satisfactoria. Así, se proporciona al desarrollador opciones que se determinarán en base a un análisis técnico de la metodología que mejor se adapte a las necesidades que se desean solventar (Laínez Fuentes, 2015) y (Zumba Gamboa \& León Arreaga, 2018)

\section{Framework}

Según Peláez Recios (2015) los framework son herramientas conformadas por módulos debidamente estructurados que son útiles en la automatización de secciones de código durante el proceso de desarrollo de software. La evolución de los framework ha dado origen al Object Relational Mapping (ORM) que son herramientas que se vinculan a una base de datos y proveen de varias funciones para extraer y transformar objetos que son modelados desde la aplicación para una correcta persistencia de datos, con lo que se reduce de forma significativa el código que deber ser introducido por el programador (Drouyer, 2015). Al aplicar estas herramientas en la academia se reduce la cantidad de elementos que el estudiante debe manipular para realizar una acción, lo que le permite asimilar de forma efectiva el conocimiento impartido.

Aplicar un ORM en la enseñanza de programación web orientado en un proyecto de software es una estrategia útil para llevar a la práctica el contenido teórico. El desarrollar un aplicativo a través de un framework ORM garantiza la reutilización de código, la aplicación de un patrón de diseño y un modelamiento estructurado lo cual influye en el tiempo y el esfuerzo que se invierte en el desarrollo del sistema.

\section{Metodología}

Se efectúo un estudio transversal sobre frameworks ORM aplicados al desarrollo de un sistema web para el control de inventario, de esta forma se analizaron las diferencias entre los productos obtenidos de los grupos de estudio (Wilmore \& Costill, 2004). Se aplicó una 
metodología cuantitativa para evaluar cada sistema y valorar el nivel de predilección, así, se logró contrastar los resultados obtenidos de cada framework. (Dominguez \& Medina, 2018)

El desarrollo del estudio se llevó a cabo con alumnos de tercer nivel de la carrera de Ingeniería en Sistemas en la materia de Programación Aplicada a la Web entre los semestres académicos abril - septiembre 2018 (periodo 1) y octubre - febrero 2019 (periodo 2) de la Universidad Laica Eloy Alfaro de Manabí Extensión en El Carmen. La población de acuerdo con Prieto Herrera (2014) es "el conjunto de medidas o el recuento de todos los elementos que representan una característica en común” (p. 112), esta se conformó por 92 alumnos divididos tal como se puede apreciar en la tabla 1, ellos fueron los encargados de desarrollar el sistema propuesto con la utilización de diferentes framework ORM. Se estableció una rúbrica para valorar el software terminado y analizar el rendimiento académico de cada grupo, adicionalmente se empleó una encuesta online para conocer la apreciación y predilección que el alumnado tiene sobre el uso de este tipo de herramientas.

Se procedió con la tabulación de los datos a través de la herramienta SPSS y con el uso de métodos como el analítico - sintético e inductivo deductivo se elaboró una interpretación de los resultados de fácil compresión.

Tabla 1.

\begin{tabular}{lll}
\hline Periodo Académico & Nivel & Alumnos \\
\hline 1 & 3ro A & 14 \\
2 & 3ro A & 38 \\
& 3ro B & 40 \\
Total & & 92 \\
\hline
\end{tabular}

Fuente: Elaboración propia

Se han seleccionado los ORM: Yii, Laravel y Zend, ya que todos aplican el lenguaje de programación PhP a través de un patrón de desarrollo Modelo Vista Controlador (MVC), el cual divide una aplicación en tres roles perfectamente diferenciados que se comunican entre sí al aplicar la programación orientada a objetos. (Ordax Cassá \& Aranzazu Ocaña, 2012) y (Honrubia López, 2014). La asignación fue realizada de forma aleatoria con el fin de mantener la imparcialidad entre los grupos, lo que estableció la siguiente distribución: Yii fue asignado al 3ro A (periodo 1), Laravel a 3ro A y Zend a 3ro B (periodo 2). Se planteó un objetivo en común bajo los requerimientos de software establecidos en la tabla 2:

Tabla 2.

\begin{tabular}{cllll}
\hline Formulario & Acciones & Puntaje & Ponderación & Total \\
\hline \multirow{4}{*}{ Categoría } & Registro de Categoría & 2,5 & & \\
& Actualización de Categoría & 2,5 & & \\
& Eliminación de Categoría & 2,5 & \multirow{2}{*}{$15 \%$} & 1,5 \\
& Validación de Datos & 2,5 & &
\end{tabular}




\begin{tabular}{|c|c|c|c|c|}
\hline Formulario & Acciones & Puntaje & Ponderación & Total \\
\hline \multirow{4}{*}{ Marca } & Registro de Marca & 2,5 & \multirow{4}{*}{$15 \%$} & \multirow{4}{*}{1,5} \\
\hline & Actualización de Marca & 2,5 & & \\
\hline & Eliminación de Marca & 2,5 & & \\
\hline & Validación de Datos & 2,5 & & \\
\hline \multirow{4}{*}{ Ítem } & Registro de Ítem & 2,5 & \multirow{4}{*}{$15 \%$} & \multirow{4}{*}{1,5} \\
\hline & Actualización de Ítem & 2,5 & & \\
\hline & Eliminación de Ítem & 2,5 & & \\
\hline & Validación de Datos & 2,5 & & \\
\hline \multirow{4}{*}{ Usuario } & Registro de Usuario & 2,5 & \multirow{4}{*}{$15 \%$} & \multirow{4}{*}{1,5} \\
\hline & Actualización de Usuario & 2,5 & & \\
\hline & Eliminación de Usuario & 2,5 & & \\
\hline & Validación de Datos & 2,5 & & \\
\hline \multirow{4}{*}{ Ingreso } & Registro de Ingreso & 2,5 & \multirow{4}{*}{$20 \%$} & \multirow{4}{*}{2} \\
\hline & Actualización de Ingreso & 2,5 & & \\
\hline & Eliminación de Ingreso & 2,5 & & \\
\hline & Validación de Datos & 2,5 & & \\
\hline \multirow{4}{*}{ Egreso } & Registro de Egreso & 2,5 & \multirow{4}{*}{$20 \%$} & \multirow{4}{*}{2} \\
\hline & Actualización de Egreso & 2,5 & & \\
\hline & Eliminación de Egreso & 2,5 & & \\
\hline & Validación de Datos & 2,5 & & \\
\hline Total: & & & $100 \%$ & 10 \\
\hline
\end{tabular}

Fuente: Elaboración propia

\section{Resultados}

Al realizar un análisis sobre las calificaciones obtenidas por los estudiantes en el desarrollo del proyecto, se puede apreciar una diferencia entre la puntuación alcanzada por cada grupo, lo cual posiciona a Zend como el framework con mayor rendimiento académico tal como se puede apreciar en la tabla 3. Se destaca que, al agrupar a los estudiantes por sexo, se evidencia una tendencia entre los tres niveles, los hombres presentan un promedio ligeramente superior al promedio obtenido por las féminas, en especial en el nivel que utiliza Laravel en donde ellas presentaron mayor dificultad, ya que este framework utiliza comandos por consola para la creación del patrón de desarrollo MVC.

Tabla 3.

\begin{tabular}{|c|c|c|c|c|c|c|}
\hline Periodo & Nivel & Framework & Sexo & Cantidad & Promedio Nivel & Promedio General \\
\hline \multirow{2}{*}{$2018(1)$} & \multirow{2}{*}{ 3ro A } & \multirow{2}{*}{ Yii } & Mujeres & 6 & 7,36 & \multirow{2}{*}{7,50} \\
\hline & & & Hombres & 8 & 7,63 & \\
\hline \multirow{3}{*}{$2018(2)$} & \multirow{2}{*}{ 3ro A } & \multirow{2}{*}{ Laravel } & Mujeres & 18 & 7,79 & \multirow{2}{*}{7,93} \\
\hline & & & Hombres & 20 & 8,07 & \\
\hline & 3ro B & Zend & $\begin{array}{l}\text { Mujeres } \\
\text { Hombres }\end{array}$ & $\begin{array}{l}18 \\
22\end{array}$ & $\begin{array}{l}8,05 \\
8,15\end{array}$ & 8,10 \\
\hline
\end{tabular}

Fuente: Elaboración propia 
Debido a la automatización que proporcionan los ORM existe una predisposición al uso de este tipo de framework, por lo cual el 96,74\% de los alumnos está dispuesto a utilizar estas herramientas en futuros trabajos o proyectos por la facilidad con la que se elaboran los diferentes formularios, eso se debe a que la interfaz de usuario es realizada por el ORM lo que le permite al desarrollador concentrarse en la programación de comportamientos. Al efectuar la demostración del software final con cada framework, Yii se sitúa como la herramienta con menos adeptos al llegar a un nivel de aceptación del 16,30\%. Por otra parte, el 66,30\% de los encuestados opta por Laravel a pesar de que este obtuvo la segunda mejor puntación sobre el análisis de rendimiento académico. Por lo antes mencionado se indica un incremento del 60,53\% en esta herramienta, de los cuales de forma inesperada el $33,33 \%$ pertenece a las mujeres tal como se puede observar en la tabla 4.

Tabla 4.

\begin{tabular}{llllll}
\hline Framework & $\begin{array}{l}\text { Porcentaje de } \\
\text { Adeptos }\end{array}$ & Sexo & Cantidad & Incremento Por sexo & $\begin{array}{l}\text { Incremento } \\
\text { General }\end{array}$ \\
\hline \multirow{2}{*}{ Laravel } & $66,30 \%$ & Mujeres & 24 & $33,33 \%$ & $60,53 \%$ \\
& & Hombres & 37 & $85,00 \%$ & \\
Zend & \multirow{2}{*}{$17,39 \%$} & Mujeres & 10 & $-44,44 \%$ & $-62,50 \%$ \\
& \multirow{2}{*}{ Yii } & Hombres & 6 & $-72,73 \%$ & $7,14 \%$ \\
& \multirow{2}{*}{$16,30 \%$} & Mujeres & 7 & $16,67 \%$ & \\
\hline & Hombres & 8 & $0,00 \%$ & \\
\end{tabular}

Se realizó un análisis sobre los factores técnicos que motivan a un estudiante el utilizar este tipo de herramientas, con lo cual se determina que la facilidad para utilizar el ORM es el criterio más importante al momento de considerar su implementación. Se muestra además que el factor que menor relevancia es el mantenimiento o actualización, ya que esta se ve equilibrada por la facilidad de manejo lo que no supone una inversión de tiempo y esfuerzo excesiva.

Tabla 5 .

\begin{tabular}{llllll}
\hline Criterio & $\begin{array}{c}\text { Muy } \\
\text { Importante }\end{array}$ & Importante & $\begin{array}{c}\text { Medianamente } \\
\text { Importante }\end{array}$ & $\begin{array}{c}\text { Nada } \\
\text { Importante }\end{array}$ & Total \\
\hline Facilidad de Uso & 46 & 30 & 13 & 3 & 92 \\
& $50,00 \%$ & $32,61 \%$ & $14,13 \%$ & $3,26 \%$ & $100 \%$ \\
Vinculación con & 29 & 45 & 11 & 7 & 92 \\
Base de Datos & $31,52 \%$ & $48,91 \%$ & $11,96 \%$ & $7,61 \%$ & $100 \%$ \\
Mantenimiento & 41 & 24 & 15 & 12 & 92 \\
& $44,57 \%$ & $26,09 \%$ & $16,30 \%$ & $13,04 \%$ & $100 \%$ \\
Nivel Seguridad & 37 & 31 & 21 & 3 & 92 \\
& $40,22 \%$ & $33,70 \%$ & $22,83 \%$ & $3,26 \%$ & $100 \%$ \\
\hline
\end{tabular}

Fuente: Elaboración propia 


\section{Conclusiones}

Los framework ORM al ser una herramienta de desarrollo con funciones prediseñadas demuestran su utilidad en la construcción de software de una forma sencilla, al ser aplicados en la academia se ha demostrado que entre los framework analizados, Zend es el que mejor comprensión de la metodología presenta y se ve reflejado en el rendimiento académico de los estudiantes. El conseguir culminar un proyecto de desarrollo de software puede generar un factor motivacional en el estudiante, al no verse frustrado ya que consigue plasmar con éxito los requerimientos solicitados dentro del tiempo estimado.

El proponer un ejercicio práctico en donde se aplique el conocimiento teórico adquirido mejora el desarrollo cognitivo, esto a su vez potencializa el desarrollo integral del estudiante al brindarle herramientas tecnológicas que estimulen al alumno a alcanzar un desarrollo óptimo de sus competencias y habilidades. Al no limitar las acciones o decisiones que el alumno pueda tomar es primordial que la información brindada por el docente sea relevante y precisa, de esta forma se evitan confusiones y se garantiza que el conocimiento adquirido sea el esperado.

La utilización de una metodología ágil de desarrollo de software se vincula perfectamente con los framework ORM, ya que se reduce el trabajo documental debido a que la herramienta genera secciones de código y dota al proyecto de flexibilidad. En el entorno de desarrollo el estudiante es capaz de adaptarse a cambios dentro del sistema lo que no modifica la planificación inicial al mantener una participación colaborativa de sus integrantes, por otra parte, una metodología más simple reduce el estrés sobre el estudiante, lo que no se lograría conseguir con métodos tradicionales.

En primera instancia el framework Zend se posiciona como la herramienta indicada para utilizarse en la cátedra de programación web, sin embargo, Laravel se muestra como la herramienta predilecta por el alumnado, a futuro se puede efectuar un análisis de estos framework ORM aplicados desde las empresas de desarrollo de software del sector para valorar el nivel de utilidad dentro de la zona.

\section{Bibliografía}

Bonvecchio, M. (2006). Evaluacion de Los Aprendizajes. Buenos Aires: Noveduc Libros.

Centeno Herrera, M. C. (2018). La intencionalidad en el desarrollo integral de la práctica profesional de los universitarios. Editorial Digital UNID.

Costa, J. L. (2015). Aprendizaje y Rendimiento Académico. Alicante: Club Universitario.

Dionicio, A. W. (2014). Intervenciones pedagógicas con b-lerning (presencial - aulas virtuales). Buenos Aires: Dunken.

Dominguez, M. C., \& Medina, M. d. (2018). Metodología de investigación para la educación y la diversidad. Madrid, España: UNED.

Drouyer, S. (2015). FuelPHP Application Development Blueprints. Birmigham, Inglaterra: Packt Publishing Ltd.

Honrubia López, F. J. (2014). Programación de aplicaciones para Iphone y Ipad. Ministerio de Educación de España. 
Laínez Fuentes, J. R. (2015). Desarrollo de Software Ágil: Extremme Programming y Scrum. (I. C. Academy, Ed.) IT Campus Academy.

Mendoza, D. (Septiembre de 2018). Influencias cognoscitivas de la tecnología de información y comunicación en el aprendizaje de la matemática. INNOVA Research Journal, 1-17. doi:https://doi.org/10.33890/innova.v3.n9.2018.584

Ordax Cassá, J. M., \& Aranzazu Ocaña, P. (2012). Programación web en Java. Ministerio de Educación de España.

Orozco, A. B., Torres, H. J., Garcia, S., Gutierrez, i. M., Acosta, L. E., Becerra, R., \& Martinez, R. (2017). Narrativa digital. Experiencias y propuestas. UNID.

Pantaleo, G., \& Rinaudo, L. (2015). Ingeniería de Software. Buenos Aires, Argentina: Alfaomega Grupo Editor.

Peláez Recios, M. L. (2015). Administración del Sistema Operativo en Sistemas ERP-CRM. Editorial Elearning, S.L.

Prieto Herrera, J. E. (2013). Investigación de mercados (Segunda ed.). Bogotá: ECOE Ediciones.

Ruiz, M., \& Aguirre, G. (octubre de 2013). Quehacer docente, TIC y educación virtual o a distancia. Apertura. Revista de Innovacion Educativa, V(2), 108-123.

Wilmore, J. H., \& Costill, D. (2004). Fisiología del esfuerzo y del deporte (Quinta ed.). Barcelona, España: Editorial Paidotribo.

Zumba Gamboa, J. P., \& León Arreaga, C. A. (Octubre de 2018). Evolución de las Metodologías y Modelos utilizados en el Desarrollo de Software. INNOVA Research Journal, 20-33. doi:https://doi.org/10.33890/innova.v3.n10.2018.651 